\title{
Introduction of IgM testing for the diagnosis of acute Lyme borreliosis: a study of the benefits, limitations and costs
}

\author{
Greg Joyner $^{1} \cdot$ Sally Mavin ${ }^{1} \cdot$ Rachel Milner $^{1} \cdot$ Chin Lim $^{1}$
}

Received: 29 July 2021 / Accepted: 11 October 2021 / Published online: 28 January 2022

(c) The Author(s) 2021

\begin{abstract}
Testing for IgM antibodies to Borrelia burgdorferi in Scottish patients with suspected Lyme borreliosis was introduced in 2018 to supplement the IgG testing already in situ. Results from 2018 to 2020 were assessed alongside available clinical data to evaluate the utility of IgM testing in serum. An estimated false positive rate of $25.5 \%$ was observed with IgM immunoblot vs $80.1 \%$ for IgM chemiluminescent immunoassay (CLIA). IgM testing can aid earlier diagnoses if used within a selective two-tier testing protocol: only patients with acute onset of symptoms should be tested for IgM CLIA but confirmation by immunoblot and consideration of clinical picture is necessary.
\end{abstract}

Keywords Serology $\cdot$ Lyme borreliosis $\cdot$ Borrelia $\cdot$ Diagnostics $\cdot \operatorname{IgM}$

\section{Introduction}

Serological testing is recommended for all suspected cases of Lyme borreliosis (LB) other than those with clinically identified erythema migrans (EM) rash. Until recently, testing for LB in Scotland utilised a two-tier testing protocol: a screening enzyme-linked immunosorbent assay (ELISA) for the detection of $\mathrm{IgG}$ antibodies to Borrelia burgdorferi sensu lato (hereafter B. burgdorferi) followed by IgG immunoblot [1]. To aid earlier detection of LB and to comply with the National Institute for Clinical Excellence (NICE) guidelines for Lyme disease published in 2018 [2], immunoblot for the detection of IgM antibodies to $B$. burgdorferi was introduced in 2018, followed by the introduction of IgM (and IgG) chemiluminescent immunoassay (CLIA) in 2020.

Methods to detect IgG antibodies to B. burgdorferi lack sensitivity during early disease, and their persistence in serum can complicate interpretation [3-8]. Although IgM antibodies are produced earlier than IgG, studies found that IgM tests have suboptimal specificity with high false positive rates due to cross-reactions with other infections and autoantibodies [9-11]. IgM may also persist [12, 13].

Sally Mavin

smirl@nhs.scot

1 Scottish Lyme Disease and Tick-Borne Infections Reference Laboratory (SLDTRL), Raigmore Hospital, Inverness IV2 3UJ, UK
The aim of this study was to evaluate the benefits and limitations of both CLIA and immunoblot for the detection of IgM antibodies to B. burgdorferi for the laboratory diagnosis of patients with acute LB.

\section{Methods}

Data for serum samples sent from throughout Scotland and tested at the Scottish Lyme Disease and Tick-Borne Infections Reference Laboratory (SLDTRL) for B. burgdorferi antibodies from 1 June 2018 to 17 October 2020 were analysed:

(i) Sera from $01 / 06 / 2018$ to $14 / 04 / 2020$ were tested by Enzygnost Lyme-link VlsE/IgG ELISA (Siemens) on the DS2 platform (Launch) following the manufacturer's instructions. Equivocal or positive sera were subsequently tested by Borrelia recomLine IgG and IgM immunoblot (Mikrogen) on the CarL immunoblot platform (Mikrogen) and the results interpreted as per the manufacturer's instructions. Samples that were $\operatorname{IgM}$ immunoblot positive and $\operatorname{IgG}$ immunoblot negative or equivocal were identified for further analyses.

(ii) Sera from $15 / 04 / 2020$ to $17 / 10 / 2020$ were tested by DiaSorin Borrelia IgG and IgM Quant CLIA on the Liaison XL analyser following discontinuation of the Enzygnost ELISA. Any samples that were positive or 
equivocal by either assay were tested by $\operatorname{IgG}$ and IgM immunoblot as above. Samples that were IgG CLIA negative and IgM CLIA reactive (positive/equivocal), IgM immunoblot positive and IgG immunoblot negative or equivocal were identified for further analyses.

Clinical information from specimen request forms, additional information from questionnaires returned from the referring clinician and any data from subsequent samples were used to allocate individual patients with isolated IgM results into groups based on likelihood of acute LB (Table 1).

\section{Results}

Of the 15,294 sera tested for LB for the period 1 June 2018 to 14 April 2020, 1304 (8.5\%) were reactive by IgG ELISA and thus tested by $\mathrm{IgG}$ and $\mathrm{IgM}$ immunoblot. Of these, 188/1304 (14.4\%) were IgM immunoblot positive and $\mathrm{IgG}$ negative or equivocal. These 188 sera came from 152 individual patients: 76 (50.0\%) were classed as "Probable" acute LB, 34 (22.4\%) as "Possible" and $30(19.7 \%)$ as "Not consistent" with acute LB. Twelve (7.9\%) patients had insufficient clinical details to assign a presumptive diagnosis (Fig. 1).

Of the 2895 sera tested for LB for the period 15 April 2020 to 17 October 2020, 661 (22.8\%) were reactive by $\operatorname{IgG}$ and/or IgM CLIA and subsequently tested by IgG and IgM immunoblot. Of these, 346 (52.3\%) were reactive for IgM CLIA only: the majority of which $(73.1 \%)$ did not confirm by immunoblot, the remaining $93(26.9 \%)$ sera were positive for IgM immunoblot only. These were from 73 individual patients: 23 (31.5\%) patients were classed as "Probable" acute LB, 24 (32.9\%) as "Possible" and 20 (27.4\%) as "Not consistent" with acute LB. Nine patients (12.3\%) had insufficient clinical details to assign a presumptive diagnosis (Fig. 2).
During the first study period (22.5 months), following the introduction of IgM immunoblot, a total of $1304 \mathrm{IgM}$ immunoblots were performed, representing an increase in laboratory consumables costs of $£ 34,556$ ( $£ 18,430$ per year). During the second study period (6 months), following the introduction of IgM CLIA, 2895 specimens were tested by IgM CLIA at an additional cost of $£ 10,595$. An additional 346 sera were tested by immunoblot as they had a reactive IgM CLIA, resulting in additional immunoblot costs of $£ 18,338$. Thus, the increase in laboratory costs after the introduction of IgM CLIA could be extrapolated to $£ 57,866$ per year.

\section{Discussion}

This study has shown that IgM immunoblot is a valuable tool in the laboratory diagnosis of LB, allowing us to detect 110 patients with "Probable" or "Possible" acute LB over the initial 22.5-month study period that may otherwise have been missed with IgG immunoblot alone. Although false positive results were obtained with the IgM immunoblot, the estimated false positive rate of $48 / 188(25.5 \%)$ was slightly lower than other recent studies [14, 15]. Introduction of IgM immunoblotting led to an increase in test costs of approximately $£ 34,556$. However, if more cases of acute LB are detected and treated early, significant cost savings to the health service could result. The increased risk of developing disseminated and late LB, along with the associated manifestations, in untreated patients has been well described [16-18]. Whilst repeat samples are routinely requested in patients with negative serology and recent onset, there is a clear potential for cases to be missed from follow-up. A 2010 study in the Netherlands found that the mean cost of disseminated LB and Lyme-related persisting symptoms was around 5700 Euros per case [19]. Although unlikely, if all 110 of the above patients were missed and progressed to disseminated LB/persisting symptoms, this could equate to 627,000 Euros as well as a huge personal cost.

Table 1 Patient groups and selection criteria for samples with isolated B. burgdorferi IgM results

\begin{tabular}{|c|c|}
\hline Patient group & Allocation criteria \\
\hline Probable acute LB & $\begin{array}{l}\text { - Clinical history/symptoms: EM rash, tick bite and rash or specific neurological symp- } \\
\text { toms, i.e. facial palsy } \\
\text { - Onset }<10 \text { weeks } \\
\text { - Follow-up sample consistent with LB }\end{array}$ \\
\hline Possible acute LB & $\begin{array}{l}\text { - Clinical history/symptoms: tick bite/exposure and flu-like symptoms } \\
\text { - Onset < } 10 \text { weeks }\end{array}$ \\
\hline Not consistent with acute LB & $\begin{array}{l}\text { - Non-specific symptoms and/or not thought to be LB by a clinician (via questionnaires) } \\
\text { - Symptoms of late LB, i.e. monoarthritis } \\
\text { - Onset }>10 \text { weeks }\end{array}$ \\
\hline Insufficient clinical details and data & Insufficient or no clinical details \\
\hline
\end{tabular}


Fig. 1 Flow diagram showing the distribution of immunoblot results (1 June 2018 to 14 April 2020) and the allocation of isolated IgM immunoblot patients into the four groups based on the likelihood of acute Lyme borreliosis (LB)

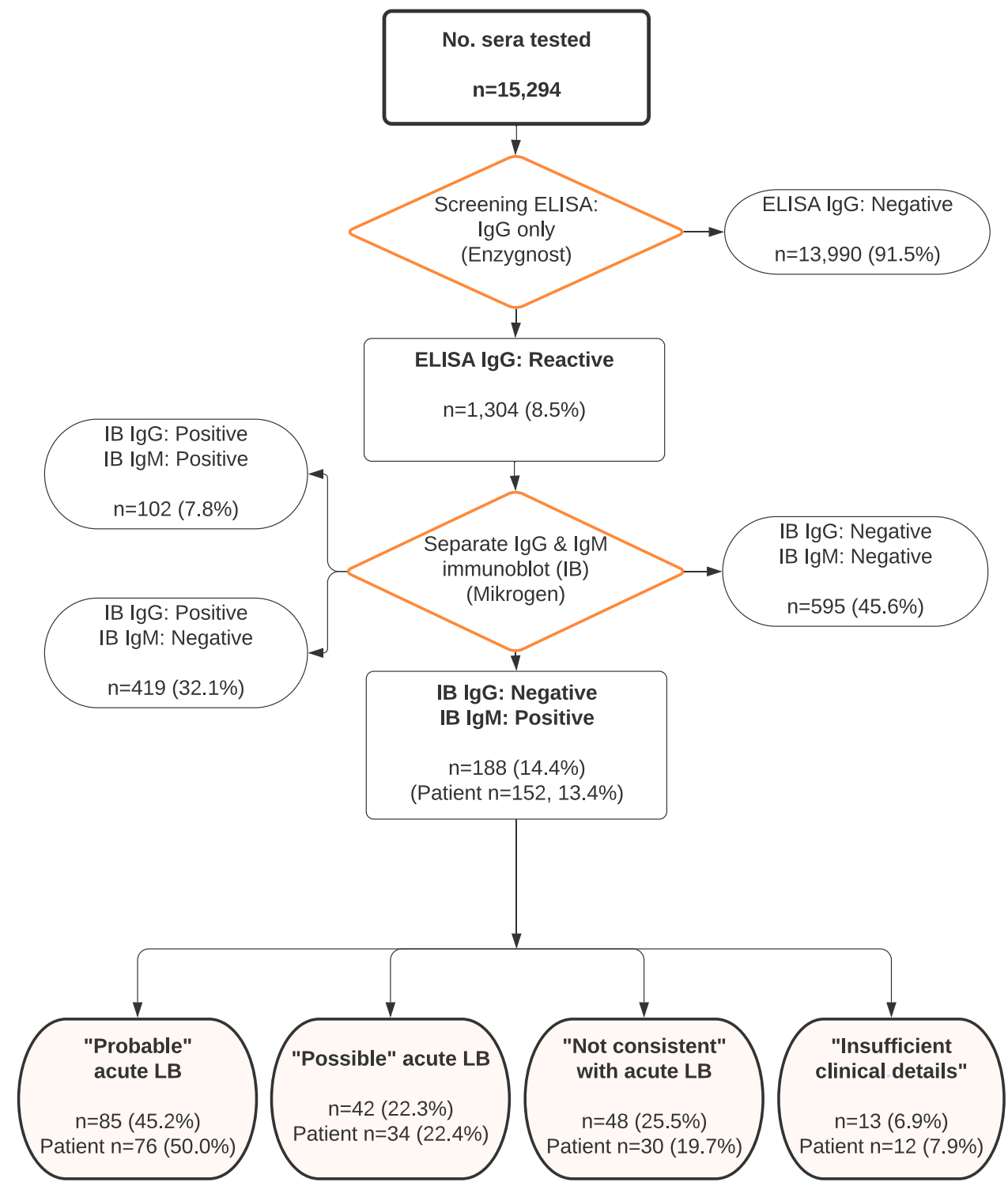

Introduction of the IgM CLIA produced a much higher false positive rate. Of the 346 sera with an isolated $\operatorname{IgM}$ CLIA result, 277 (80.1\%) were potentially false positive as they either did not confirm by immunoblot or were assessed as "Not consistent" with acute LB. This highlights that IgM CLIA testing in sera should only be used as part of a robust two-tiered testing protocol with confirmatory testing. The high false positive rate obtained for the IgM CLIA meant that a much higher proportion of samples required immunoblot testing: $22.9 \%$ of sera tested by CLIA (April to October 2020) were reactive with $\mathrm{IgG}$ and/or IgM CLIA, in contrast with the $8.5 \%$ of sera that were reactive with the IgG ELISA in the previous study period. This put extra pressure on the laboratory staff and greatly increased test costs (£28,933). It could be argued that the benefits of IgM CLIA are only marginal and perhaps not cost-efficient; however, 47 patients with probable or possible acute LB were detected, which may otherwise have been missed. Again, although unlikely, if all 47 were missed and progressed to disseminated LB/ persisting symptoms, this could equate to 267,900 Euros.

Our results show that there is a significant risk of reporting of inaccurate and misleading results if patients are diagnosed on the basis of an isolated $\operatorname{IgM}$ positive result without consideration of clinical details, disease duration and pre-test probability. Prior studies have found that the majority of patients tested for Lyme serology did not meet European or UK clinical case definitions, and recommend that pre-test probability of infection is considered $[5,6,20]$. As a degree of seroprevalence for B. burgdorferi-specific antibodies exists in the population, over testing can lead to high false positive rates. The cost of misdiagnosing someone with acute LB based on false positive results, leading to their inappropriate, ineffective or even harmful treatment with 
Fig. 2 Flow diagram showing the distribution of CLIA results (15 April 2018 to 17 October 2020) and the allocation of positive IgM CLIA confirmed by IgM immunoblot samples and patients into the four groups based on the likelihood of acute Lyme borreliosis (LB)

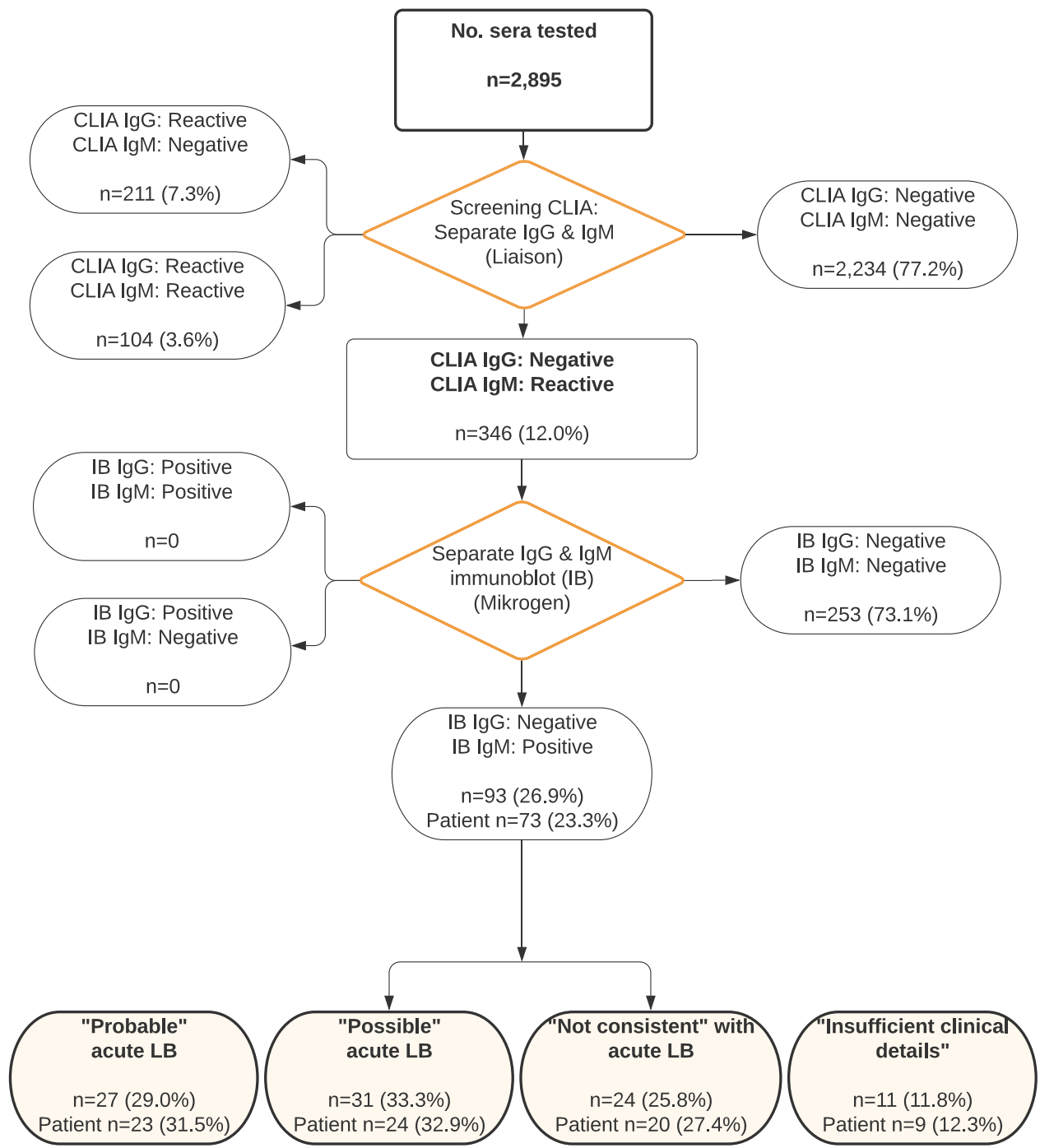

antibiotics, and potentially delaying further investigations into the cause of their symptoms, should not be ignored.

The authors concede that the categorisation of patients into "Probable" or "Possible" LB in this study was flawed as there was limited clinical information available. For this reason, assay sensitivity, specificity and positive predictive values could also not be assessed. Many of the samples tested were not accompanied with adequate clinical details such as symptoms and date of onset. The authors accept this may have influenced the study false positive rate and whilst this may indicate that our testing protocols are suboptimal, this is the real-world scenario for a large number of laboratories where demand for Lyme testing is high and illustrates the challenges faced when interpreting results. The authors also recognise that the study results may have been influenced by the actual assays utilised. It is widely recognised that $B$. burgdorferi assays lack inter-assay consensus, particularly IgM assays [21, 22]. Interestingly, one of these studies calculated that a small loss of specificity led to an additional
192,716 immunoblot tests required (4,625,183 Euros), with an additional $6191 \mathrm{IgM}$ false positive results.

Due to the issues outlined above, some countries have, or are considering, stopping the use of IgM testing for LB. However, some cases of early disease may be missed and patient confidence in testing regimes will undoubtedly be affected, which could further fuel the controversy around testing. Some manufacturers claim that IgG antibodies to the VlsE antigen of B. burgdorferi can be detected prior to or parallel to the formation of $\operatorname{IgM}$ antibodies and are more specific than IgM assays; thus, the use of VlsE/IgG screening assays is sufficient. However, the IgG CLIA used in this study, which contains the VlsE antigen, missed some cases of acute LB. Although complex to implement in laboratories with a high throughput of specimens, perhaps the use of selective testing protocols would be optimal, utilising $\operatorname{IgM}$ CLIA only for those patients with an acute onset of specific symptoms within a two-tier testing protocol. 
Author contribution GJ: testing, data analysis and original draft preparation. SM: conceptualization, supervision, review, editing and redraft preparation. RM: testing, review and editing. CL: review and editing, clinical expertise.

Funding This research was supported as part of Northtick, an Interreg project supported by the North Sea Programme of the European Regional Development Fund of the European Union. SM is a member of ESGBOR.

\section{Data availability N/A}

Code availability N/A

\section{Declarations}

\section{Ethics approval N/A}

\section{Consent to participate N/A}

\section{Consent for publication N/A}

Conflict of interest The authors declare no competing interests.

Open Access This article is licensed under a Creative Commons Attribution 4.0 International License, which permits use, sharing, adaptation, distribution and reproduction in any medium or format, as long as you give appropriate credit to the original author(s) and the source, provide a link to the Creative Commons licence, and indicate if changes were made. The images or other third party material in this article are included in the article's Creative Commons licence, unless indicated otherwise in a credit line to the material. If material is not included in the article's Creative Commons licence and your intended use is not permitted by statutory regulation or exceeds the permitted use, you will need to obtain permission directly from the copyright holder. To view a copy of this licence, visit http://creativecommons.org/licenses/by/4.0/.

\section{References}

1. Centers for Disease Control and Prevention (CDC) (1995) Recommendations for test performance and interpretation from the Second National Conference on Serologic Diagnosis of Lyme Disease. MMWR Morb Mortal Wkly Rep 44(31):590-591. https:// doi.org/10.1001/jama.1995.03530120023018

2. National Guideline Centre (UK) (2018) Lyme disease National Institute for Health and Care Excellence (NICE). NBK542111 https://www.nice.org.uk/guidance/ng95. Accessed 26 Jul 2021

3. Munro H, Mavin S, Duffy K et al (2015) Seroprevalence of Lyme borreliosis in Scottish blood donors. Transfus Med 25(4):284286. https://doi.org/10.1111/tme.12197

4. Bušová A, Dorko E, Rimárová K et al (2018) Seroprevalence of Lyme disease in Eastern Slovakia. Cent Eur J Public Health 26(88):S67-S71. https://doi.org/10.21101/cejph.a5442

5. Dessau RB, van Dam AP, Fingerle V et al (2018) To test or not to test? Laboratory support for the diagnosis of Lyme borreliosis: a position paper of ESGBOR, the ESCMID study group for Lyme borreliosis. Clin Microbiol Infect 24(2):118-124. https://doi.org/ 10.1016/j.cmi.2017.08.025

6. Hillerdal H, Henningsson AJ (2021) Serodiagnosis of Lyme borreliosis-is IgM in serum more harmful than helpful? Eur J Clin Microbiol Infect Dis. https://doi.org/10.1007/s10096-020-04093-2
7. Huycke MM, D’Alessio DD, Marx JJ (1992) Prevalence of antibody to Borrelia burgdorferi by indirect fluorescent antibody assay, ELISA, and Western immunoblot in healthy adults in Wisconsin and Arizona. J Infect Dis 165(6):1133-1137. https://doi. org/10.1093/infdis/165.6.1133

8. Strizova Z, Smrz D, Bartunkova J (2020) Seroprevalence of Borrelia IgM and $\mathrm{IgG}$ antibodies in healthy individuals: a caution against serology misinterpretations and unnecessary antibiotic treatments. Vector Borne Zoonotic Dis 20(10):800-802. https:// doi.org/10.1089/vbz.2020.2632

9. Golkocheva-Markova E, Christova I, Stoilov R et al (2008) Cross-reaction between Yersinia outer membrane proteins and anti-Borrelia antibodies in sera of patients with Lyme disease. Clin Microbiol Infect 14(9):873-875. https://doi.org/10.1111/j. 1469-0691.2008.02051.x

10. Magnarelli LA, Anderson JF, Johnson RC (1987) Cross-reactivity in serological tests for Lyme disease and other spirochetal infections. J Infect Dis 156(1):183-188. https://doi.org/10.1093/infdis/ 156.1.183

11. Lovece S, Stern R, Kagen LJ (1991) Effects of rheumatoid factor, antinuclear antibodies and plasma reagin on the serologic assay for Lyme disease. J Rheumatol 18(12):1813-1818

12. Murray KO, Garcia MN, Yan C et al (2013) Persistence of detectable immunoglobulin $\mathrm{M}$ antibodies up to 8 years after infection with West Nile virus. Am J Trop Med Hyg 5:996-1000. https:// doi.org/10.4269/ajtmh.13-0232

13. Kalish RA, McHugh G, Granquist J et al (2001) Persistence of immunoglobulin $\mathrm{M}$ or immunoglobulin $\mathrm{G}$ antibody responses to Borrelia burgdorferi 10-20 years after active Lyme disease. Clin Infect Dis 33(6):780-785. https://doi.org/10.1086/322669

14. Lantos PM, Lipsett SC, Nigrovic LE (2016) False positive Lyme disease IgM immunoblots in children. J Pediatr 174:267-269.e1. https://doi.org/10.1016/j.jpeds.2016.04.004

15. Webber BJ, Burganowski RP, Colton L et al (2019) Lyme disease overdiagnosis in a large healthcare system: a population-based, retrospective study. Clin Microbiol Infect 25(10):1233-1238. https://doi.org/10.1016/j.cmi.2019.02.020

16. Bratton RL, Whiteside JW, Hovan MJ et al (2008) Diagnosis and treatment of Lyme disease. Mayo Clin Proc 83(5):566-571. https://doi.org/10.4065/83.5.566

17. Hengge UR, Tannapfel A, Tyring SK et al (2003) Lyme borreliosis. Lancet Infect Dis 3(8):489-500. https://doi.org/10.1016/ s1473-3099(03)00722-9

18 Hatchette TF, Davis I, Johnston BL (2014) Lyme disease: clinical diagnosis and treatment. Can Commun Dis Rep 40(11):194-208. https://doi.org/10.14745/ccdr.v40i11a01

19. van den Wijngaard CC, Hofhuis A, Wong A et al (2017) The cost of Lyme borreliosis. Eur J Public Health 27(3):538-547. https:// doi.org/10.1093/eurpub/ckw269

20. John TM, Taege AJ (2019) Appropriate laboratory testing in Lyme disease. Cleve Clin J Med 86(11):751-759. https://doi.org/10. 3949/ccjm.86a.19029

21. Lager M, Dessau RB, Wilhelmsson P et al (2019) Serological diagnostics of Lyme borreliosis: comparison of assays in twelve clinical laboratories in Northern Europe. Eur J Clin Microbiol Infect Dis 38(10):1933-1945. https://doi.org/10.1007/ s10096-019-03631-x

22. Müller I, Freitag MH, Poggensee G et al (2012) Evaluating frequency, diagnostic quality, and cost of Lyme borreliosis testing in Germany: a retrospective model analysis. Clin Dev Immunol 2012:595427. https://doi.org/10.1155/2012/595427

Publisher's note Springer Nature remains neutral with regard to jurisdictional claims in published maps and institutional affiliations. 